\title{
ARIADNE, a Photographic Two-Phase LAr TPC
}

\author{
Adam Roberts*, J. Carroll, K. Majumdar, K. Mavrokoridis, K. J. McCormick, B. \\ Philippou, N. A. Smith, G. Stavrakis, C. Touramanis, J. Vann \\ University of Liverpool (GB) \\ Department of Physics, Oliver Lodge Bld, Oxford Street, Liverpool, L69 7ZE, UK \\ E-mail: aroberts@hep.ph.1iv.ac.uk
}

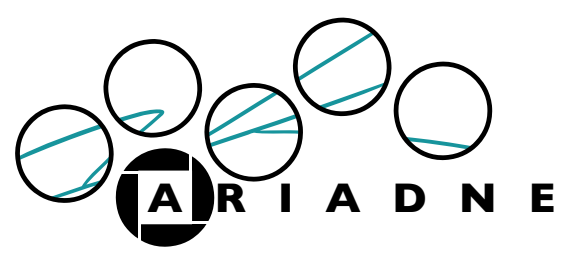

\begin{abstract}
There is a significant $\mathrm{R} \& \mathrm{D}$ effort ongoing in the liquid argon (LAr) time projection chamber (TPC) community. Due to the scintillation and ionisation properties of LAr, coupled with its low cost, it is possible to construct detectors with immense fiducial volume at a modest cost. It is for this reason that LAr TPCs are fast becoming the detector of choice for long baseline neutrino physics experiments. Using LAr TPCs it is possible to provide the large targets required to make direct observation of neutrino interactions a statistically reasonable prospect. Experiments such as the deep underground neutrino experiment (DUNE) are proposing the use of 68,000 tons of liquid argon as the target material of choice shared between four colossal LAr TPCs. In a twophase TPC the charge amplification process is performed in the gas phase. By performing charge amplification in the gas phase it is possible to achieve much higher gains and hence improved signal-to-noise ratios. ARIADNE is a novel and innovative two-phase LAr TPC pioneering the direct imaging of secondary scintillation light produced during the charge amplification process using a THGEM in the gas phase. Optical readout presents many advantages over current readout technologies such as massive reductions in the number of readout channels, ease of scalability, upgrade, installation and maintenance as well as cost effectiveness.
\end{abstract}

ARIADNE is an ERC funded experiment, grant number 677927.

The European Physical Society Conference on High Energy Physics

5-12 July

Venice, Italy

${ }^{*}$ Speaker. 


\section{The ARIADNE experiment}

The goal of ARIADNE is to mature the optical readout technology first demonstrated at Liverpool [1]. ARIADNE will be deployed to a charged particle beamline where the ability to perform particle identification (PID), electron-gamma separation, cross section studies plus more will be investigated. The outcome of this experiment has the potential to inform the design of future LAr TPCs. Figure 1a shows the TPC optical readout detection principle while Figure $1 \mathrm{~b}$ presents an image of a cosmic particle captured using the 40-litre prototype detector.
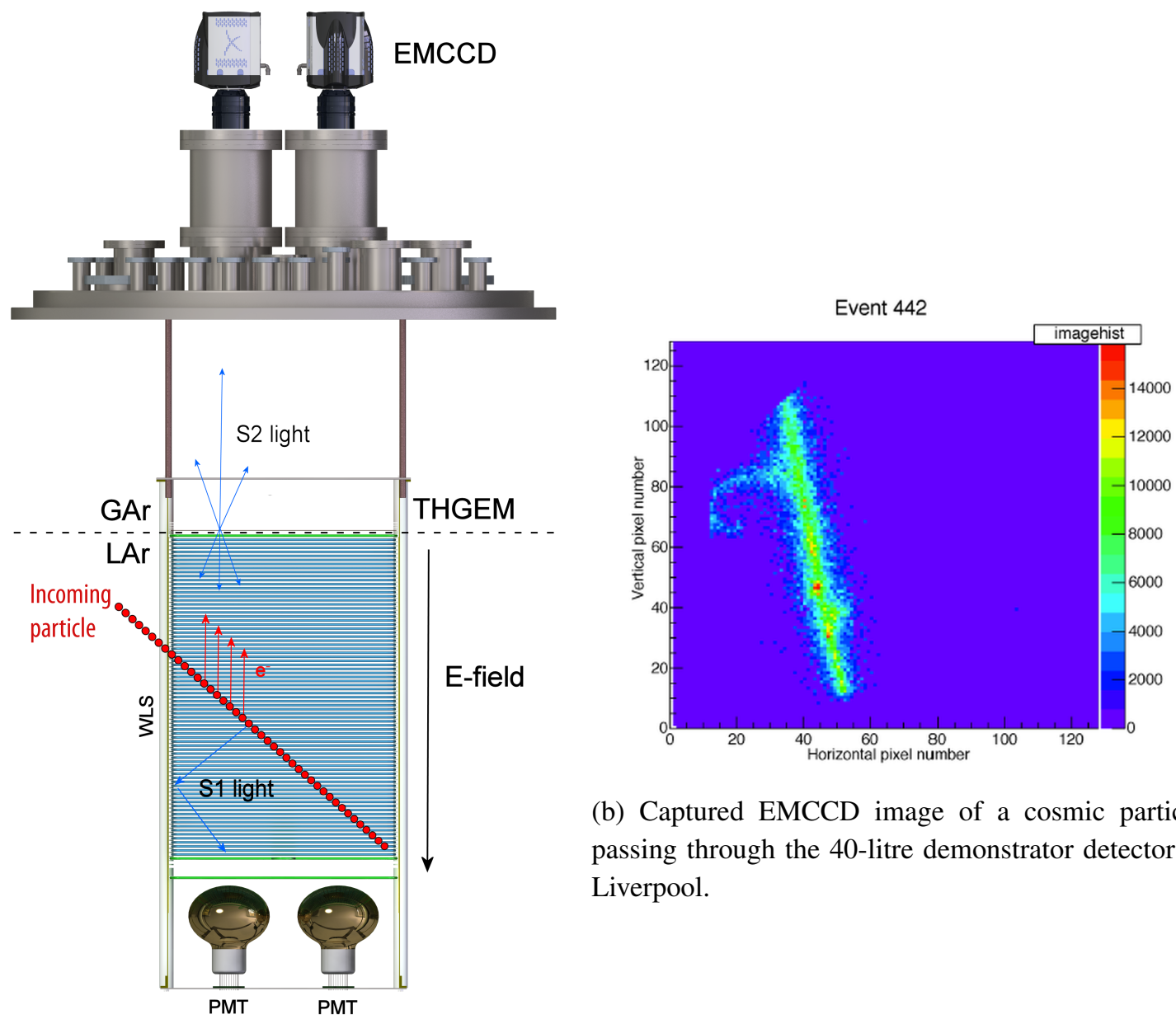

(b) Captured EMCCD image of a cosmic particle passing through the 40-litre demonstrator detector at Liverpool.

(a) ARIADNE operating principle. Secondary scintillation (S2) light produced in the THGEM holes during charge multiplication process can be captured using a highly sensitive EMCCD camera.

Figure 1: The ARIADNE detection principle and proof of concept. 


\section{The ARIADNE detector}

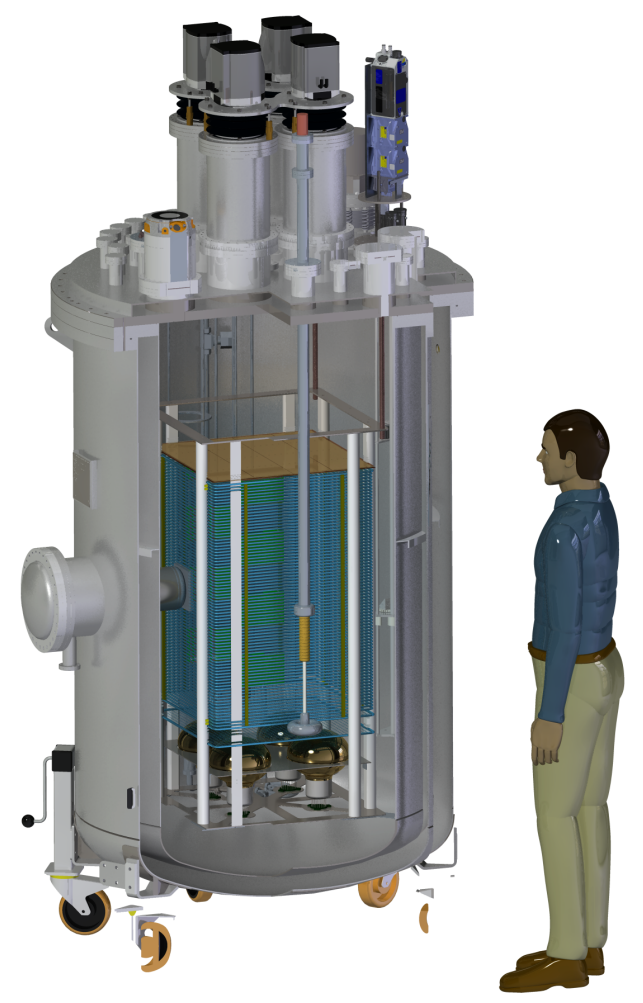

(a) An overall view of the ARIADNE experiment. The TPC is suspended in the $\oslash 1.07 \mathrm{~m} \times 2.1 \mathrm{~m}$ tall cryostat. The beam window is visible on the left side of the cryostat.

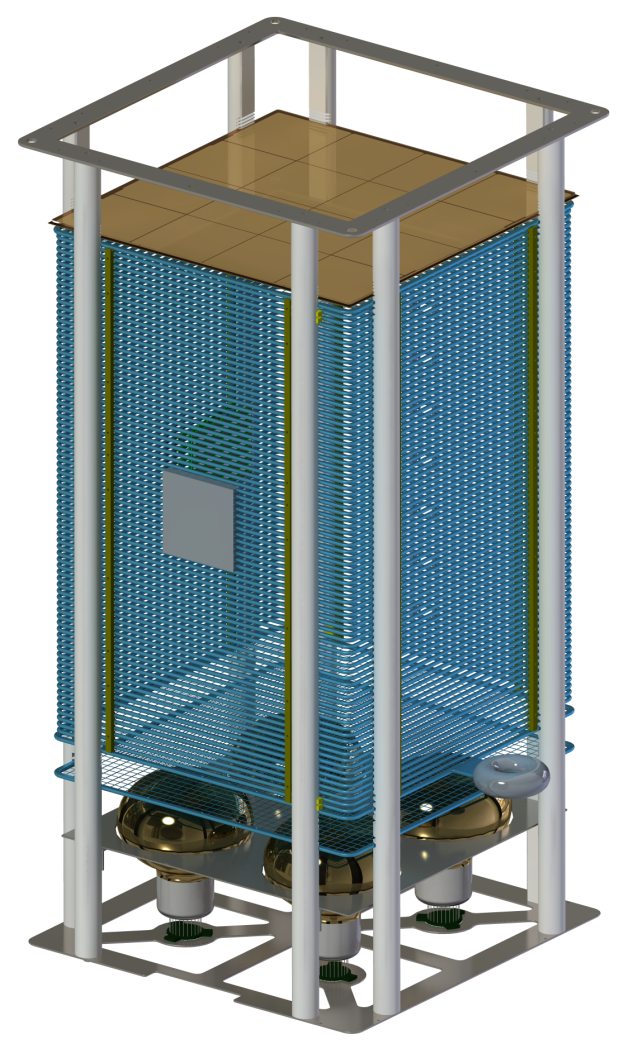

(b) The ARIADNE TPC. The fiducial volume is $54 \mathrm{x}$ $54 \times 80 \mathrm{~cm}^{3}$. The 79 field cage rings spaced $1 \mathrm{~cm}$ apart give a maximum drift length of $80 \mathrm{~cm}$.

Figure 2: The ARIADNE detector

The heart of the ARIADNE detector is a $54 \times 54 \times 80 \mathrm{~cm}^{3}$ fiducial volume TPC which is housed inside of a purpose-built cryostat. The ARIADNE cryostat has a beam window, visible on the left hand side of Figure 2a. This beam window allows particles to travel through vacuum until they are close to the fiducial volume, without having to travel through large distances of uninstrumented LAr. This ensures a tight momentum distribution of the particles entering the fiducial volume. A total of 79 field cage rings spaced $1 \mathrm{~cm}$ apart provide the uniform drift field inside the TPC. The cryostat is partially filled with LAr such that the THGEM remains in the gas phase while the rest of the TPC is in the liquid phase. A segmented THGEM with 16 anodes performs charge multiplication in the gas phase. Four Hamamatsu R5912-02MOD PMTs provide detector triggering on primary scintillation light (S1) as well as readout of secondary (S2) light produced during the charge multiplication process. The top flange of the cryostat has a selection of feedthroughs which allow access for high voltage supply to the TPC, vacuum pumps, a custom laser calibrations system as well as optical viewports for the EMCCD cameras. Four Andor iXon Ultra 888 EMCCD 
cameras observe light produced via electroluminescence in the holes of the THGEM via these viewports in the top flange.

\section{Outlook}

ARIADNE will be deployed to a mixed charged particle beamline $[2,3]$ in 2018. Using a beamline with a mixed particle content allows for PID selection efficiency studies. These studies will require the development of shower and track reconstruction plus particle classification algorithms. Since ARIADNE is pioneering a new type of readout, a different perspective on event topology may result, requiring the development of new analysis techniques. There is an exciting opportunity for developments involving neural networks/image classifiers to perform PID and event reconstruction. Development of these algorithms/tools may be valuable for future LAr TPC analysis efforts.

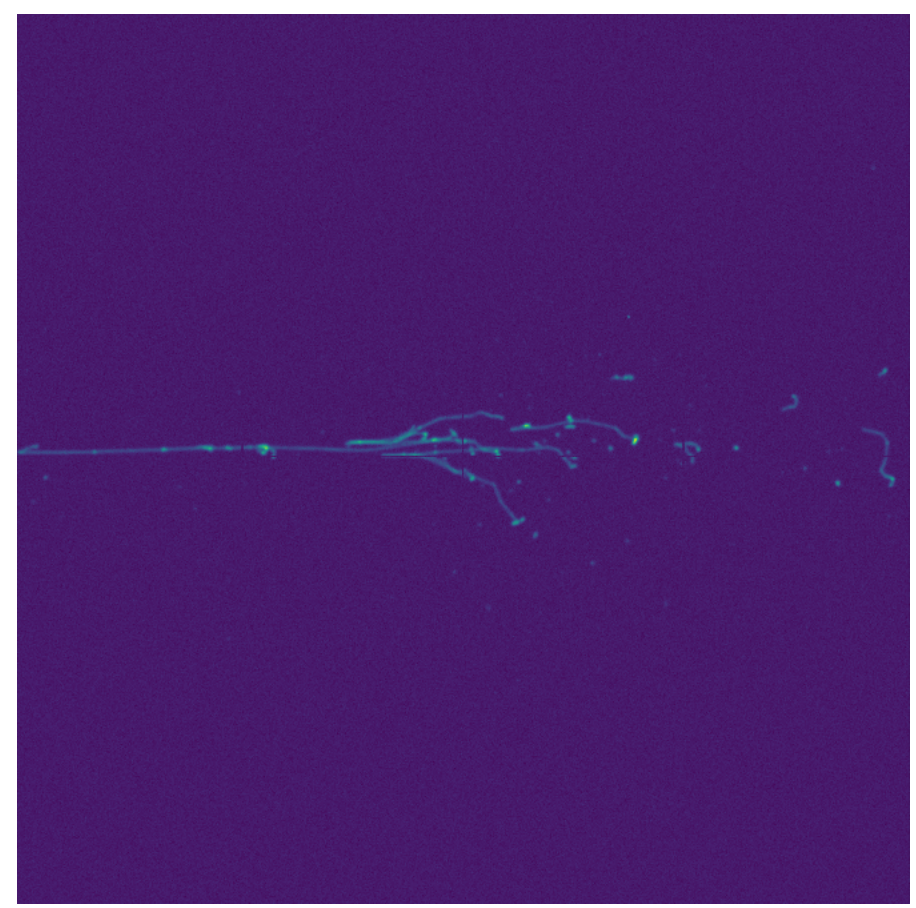

Figure 3: ARIADNE preliminary simulated interaction inside the fiducial volume using Geant 4 . A single e+ with $500 \mathrm{MeV}$ momentum enters from the left.

\section{References}

[1] K. Mavrokoridis, J. Carroll, K. J. McCormick, P. Paudyal, A. Roberts, N. A. Smith, C. Touramanis. First Demonstration of Imaging Cosmic Muons in a Two-Phase Liquid Argon TPC using an EMCCD Camera and a THGEM. https: / / arxiv.org/abs/1507.06586

[2] K. Mavrokoridis, K. J. McCormick, M. Nessi, A. Roberts, N. A. Smith, C. Touramanis Letter of Intent: ARIADNE, a Photographic LAr TPC at the CERN Neutrino Platform. https://cds.cern.ch/record/2123865/files/SPSC-I-244.pdf

[3] http://hep.ph.liv.ac.uk/ariadne/ 